\title{
A Numerical Study of the Analytical Downward Continuation Error in Geoid Computation by EGM08
}

\author{
Research Article
}

\author{
L. E. Sjöberg*, M. Bagherbandi
}

Division of Geodesy, Royal Institute of Technology, SE-100 44 Stockholm, Sweden

\begin{abstract}
:
Today the geoid can be conveniently determined by a set of high-degree spherical harmonics, such as EGM08 with a resolution of about 5 '. However, such a series will be biased when applied to the continental geoid inside the topographic masses. This error we call the analytical downward continuation (DWC) error, which is closely related with the so-called topographic potential bias. However, while the former error is the result of both analytical continuation of the potential inside the topographic masses and truncation of a series, the latter is only the effect of analytical continuation.

This study compares the two errors for EGM08, complete to degree 2160. The result shows that the topographic bias ranges from 0 at sea level to $5.15 \mathrm{~m}$ in the Himalayas region, while the DWC error ranges from $-0.08 \mathrm{~m}$ in the Pacific to $5.30 \mathrm{~m}$ in the Himalayas. The zero-degree effects of the two are the same $(5.3 \mathrm{~cm})$, while the rms of the first degree errors are both $0.3 \mathrm{~cm}$. For higher degrees the power of the topographic bias is slightly larger than that for the DWC error, and the corresponding global rms values reaches 25.6 and $25.3 \mathrm{~cm}$, respectively, at $n_{\max }=2160$. The largest difference $(20.5 \mathrm{~cm})$ was found in the Himalayas. In most cases the DWC error agrees fairly well with the topographic bias, but there is a significant difference in high mountains. The global rms difference of the two errors clearly indicates that the two series diverge, a problem most likely related with the DWC error.
\end{abstract}

\section{Keywords:}

Analytical continuation • downward continuation • topographic bias • EGM08

(c) Versita Warsaw and Springer-Verlag Berlin Heidelberg.

Received 11 November 2010; accepted 18 Novem ber 2010

1. Introduction

In 2008 the U.S. National Geospatial-Intelligence Agency (NGA) EGM Development Team released the Earth Gravitational Model EGM08, complete to degree and order 2160 (Pavlis et al. 2008). The long-wavelength part of the model was obtained from GRACE (Gravity Recovery And Climate Experiment) data to degree and order 60 , and the medium- to short-wavelengths were computed by from a global coverage of $5^{\prime} \times 5^{\prime}$ terrestrial gravity anomalies (generated by satellite altimetry over the oceans). EGM08 may

*E-mail: 1sjo@kth.se, Tel: +46-8-790-7330; Fax: +46-8-790-7343

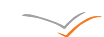

VERSITA provide estimates of the geoid height globally to an accuracy of the order of $1 \mathrm{~cm}$ at a resolution of $5^{\prime} \times 5^{\prime}$ (i.e., about $10 \times 10 \mathrm{~km}^{2}$ ). However, as for continental regions the geoid is mainly located within the topographic masses, the representation of the disturbing potential of the Earth by a series of spherical harmonics, which is the case when applying EGM08, will be biased. This is because EGM08 is related with an external type of harmonic series, while inside the topographic masses the potential cannot be harmonic. Sjöberg (1977) and (1980) as well as Jekeli (1981) studied "the downward continuation error" (DWC error) as the difference between an external and internal type of harmonic series. This error is not due only to the fact that the actual potential is not harmonic inside the topographic masses (the topographic bias), while the harmonic series is, but it is due also to other problems of the series, 
such as truncation and convergence. In Sjöberg (2007), (2008) and (2009a) the so-called topographic potential bias was clearly distinguished from the DWC error.

In a harmonic expansion of the disturbing potential to degree 16 , based on $5^{\circ} \times 5^{\circ}$ spherical blocks of mean topographic heights, Sjöberg (1977) found that the global rms DWC error at sea level, i.e. the geoid error, is about $0.13 \mathrm{~m}$, and the error coefficients were tabulated to the same degree (Sjöberg ibid., Table 3). On the contrary, Jekeli (1981) studied only the DWC error at the Earth's surface, which, of course, does not include any analytical continuation and therefore no topographic bias. He concluded that the DWC error of the quasigeoid height, based on an external type of spherical harmonics of the disturbing potential to degree and order 300, does not exceed $0.4 \mathrm{~mm}$. Ågren (2004) studied numerically the difference between the "strict" DWC error (Eq. 3 below) and that achieved by using a binomial series expansion of topographic height (Eq. 9 below). For a harmonic series to degree and order 1800 he found good agreement between the two formulas (using only binomial series to second order) when excluding the highest mountain areas. When including also terms up to power 4 in the binomial series the maximum reported difference was $16 \mathrm{~mm}$ (in the Himalayas).

The questions we will discuss in this article are the following:

1. how significant is the DWC error in applying EGM08 to geoid computation?

2. Does this error differ significantly from the topographic bias?

\section{The downward continuation error}

The external type of spherical harmonic representation of the topographic potential applied at sea level and truncated at degree $n_{\max }$ becomes (Sjöberg 1977)

$$
V_{e}^{T}(\theta, \lambda)=\mu R^{2} \sum_{n=0}^{n_{\max }} \sum_{m=-n}^{n}{ }^{e} V_{n m}^{T} Y_{n m}(\theta, \lambda)
$$

where

${ }^{e} V_{n m}^{T}=\frac{1}{(2 n+1)(n+3)} \iint_{\sigma}\left[\left(\frac{r_{S}}{R}\right)^{n+3}-1\right] Y_{n m}(\theta, \lambda) d \sigma$

Here $\mu$ is the product of the gravitational constant and topographic density (assumed to be constant), $R$ and $r_{S} \geqslant R$ are the radii of sea level (assumed to be spherical) and the topographic surface, respectively, and $\sigma$ is the unit sphere, and $Y_{n m}$ is the fullynormalized spherical harmonic of degree $n$ and order $m$ with arguments $(\theta, \lambda)$ being co-latitude and longitude.

The corresponding internal type harmonic series becomes (Sjöberg 1977, Sect. 4)

$$
V_{i}^{T}(\theta, \lambda)=\mu R^{2} \sum_{n=0}^{n_{\max }} \sum_{m=-n}^{n}{ }^{i} V_{n m}^{T} Y_{n m}(\theta, \lambda)
$$

where

$$
\begin{gathered}
{ }^{i} V_{n m}^{T}=\frac{1}{(2 n+1)(n-2)} \iint_{\sigma}\left[\left(\frac{R}{r_{S}}\right)^{n-2}-1\right] Y_{n m}(\theta, \lambda) d \sigma \\
\text { if } n \neq 2
\end{gathered}
$$

and

$$
{ }^{i} V_{2 m}^{T}=\iint_{\sigma} \ln \left(r_{S} / R\right) Y_{n m}(\theta, \lambda) d \sigma, \quad \text { if } n=2
$$

As discussed in Sjöberg (1977), (1980) and (2008) two main problems may occur when applying Eq. (1) at sea level:

1. the series will be biased within the topography (topographic bias; see Sect. 3),

2. the series may be part of a divergent series and therefore incorrect.

The bias means a systematic error committed by analytically continuing a harmonic series into the topographic masses, where the correct potential is not harmonic. The problem of convergence is not limited to the application of the series to a point inside the topographic masses, but it is relevant to any computational point below the Brillouin sphere, i.e. the limiting sphere enveloping all topographic masses. In addition, as the series is truncated, it also experiences a truncation error. In any case, while the internal series, Eq. (2), suffers only from a truncation error from the terms beyond degree $n_{\max }$, the external type series, Eq. (1), suffers from truncation, analytical continuation into masses and the possible error related with a divergent series. By taking the difference between the two series we get what we call the DWC error of the external series [but, actually, as the truncation errors differ between the two series, the DWC error is composed of errors of truncation, topographic bias and, possibly, convergence of the series]. Explicitly the geoid height error becomes (when scaled to geoid height by dividing the potential by normal gravity $\gamma$ )

$$
\begin{aligned}
& \delta N(\theta, \lambda)= \\
& \frac{\mu}{R \gamma} \sum_{n=0}^{n_{\max }} \iint_{\sigma} \int_{R}^{r_{s}}\left[\left(\frac{r}{R}\right)^{n}-\left(\frac{R}{r}\right)^{n+1}\right] r^{2} d r P_{n}(\cos \psi) d \sigma(3)
\end{aligned}
$$

where $P_{n}(\cos \psi)$ is the Legendre's polynomial of degree $n$ and $\psi$ is the geocentric angle. Except for the sign, this formula agrees with "the combined topographic effect" as presented in Sjöberg (2000, Eq. 113). After integration with respect to radius, this 
equation can also be written in terms of spherical harmonics (cf. Eqs. 1a-2c):

$$
\delta N(\theta, \lambda)=\frac{\mu R^{2}}{\gamma} \sum_{n=0}^{n_{\max }} \sum_{m=-n}^{n} A_{n m} Y_{n m}(\theta, \lambda)
$$

where

$$
A_{n m}=\frac{1}{2 n+1} \iint_{\sigma} I_{n}\left(R, r_{S}\right) Y_{n m}(\theta, \lambda) d \sigma
$$

Here (see Sjöberg 1977)

$I_{n}\left(R, r_{S}\right)= \begin{cases}\frac{\left(r_{S} / R\right)^{n+3}-1}{n+3}+\frac{\left(R / r_{S}\right)^{n-2}-1}{n-2} & \text { if } n \neq 2 \\ \frac{\left(r_{S} / R\right)^{5}-1}{5}-\ln \left(r_{S} / R\right) & \text { if } n=2\end{cases}$

Eqs. (4a), (4b) and (5) can also be obtained by directly taking the difference between the external and internal types harmonic series of Eqs. (1a) and (2a).

By inserting the binomial series expansions (with $H=r_{S}-R$ )

$$
\left(\frac{r_{S}}{R}\right)^{n+3}=\left(1+\frac{H}{R}\right)^{n+3}=\sum_{k=0}^{\infty}\left(\begin{array}{c}
n+3 \\
k
\end{array}\right)\left(\frac{H}{R}\right)^{k}
$$

and

$$
\left(\frac{r_{S}}{R}\right)^{-(n-2)}=\sum_{k=0}^{\infty}\left(\begin{array}{c}
-(n-2) \\
k
\end{array}\right)\left(\frac{H}{R}\right)^{k}
$$

into Eq. (5) we obtain also

$$
\begin{aligned}
& I_{n}\left(R, r_{S}\right)=\sum_{k=2}^{\infty}\left[\left(\begin{array}{c}
n+2 \\
k
\end{array}\right)-\left(\begin{array}{c}
-n+1 \\
k
\end{array}\right)\right]\left(\frac{H}{R}\right)^{k}= \\
& (2 n+1)\left(\frac{H}{R}\right)^{2}\left[\frac{1}{2}+\frac{1}{3} \frac{H}{R}+\frac{n(n+1)}{2 \times 3 \times 4}\left(\frac{H}{R}\right)^{2}+\ldots\right]
\end{aligned}
$$

By inserting Eq. (7) into Eq. (4b) one arrives at

$A_{n m}=2 \pi\left(\left(H^{2}\right)_{n m}+\frac{2\left(H^{3}\right)_{n m}}{3 R}+\frac{n(n+1)}{3 \times 4} \frac{\left(H^{4}\right)_{n m}}{R^{2}}+\ldots\right)$

where

$$
H_{n m}^{v}=\frac{1}{4 \pi} \iint_{\sigma} H^{v} Y_{n m}(\theta, \lambda) d \sigma,
$$

so that Eq. (4a) becomes

$$
\begin{aligned}
& \delta N(\theta, \lambda)= \\
& \frac{2 \pi \mu}{\gamma} R^{2} \sum_{n=0}^{n_{\max }} \sum_{m=-n}^{n} \sum_{k=2}^{\infty}\left[\left(\begin{array}{c}
n+2 \\
k
\end{array}\right)-\left(\begin{array}{c}
-n+1 \\
k
\end{array}\right)\right] . \\
& \cdot\left(\frac{H}{R}\right)^{k} Y_{n m}(\theta, \lambda)= \\
& \frac{2 \pi \mu}{\gamma} \sum_{n=0}^{n_{\max }} \sum_{m=-n}^{n}\left(\left(H^{2}\right)_{n m}+\frac{2\left(H^{3}\right)_{n m}}{3 R}+\frac{n(n+1)}{3 \times 4} \frac{\left(H^{4}\right)_{n m}}{R^{2}}+\ldots\right) . \\
& Y_{n m}(\theta, \lambda) .
\end{aligned}
$$

\subsection{Convergence of the series}

In the strict sense we should not expect Eq. (3) to be convergent when $n_{\max }$ approaches infinity. This is obvious, as the ratio $r_{S} / R \geqslant 1$. Nevertheless, for a finite $n_{\max }$ the series is also finite. The convergence problem holds also for the binomial series of Eq. (7); see Sun and Sjöberg (2001). In the numerical application we will only consider a finite $n_{\max }$, so that convergence will not be a problem. The convergence problem was extensively described in Moritz (1980, Chs. 6-7). Moritz (ibid.) and Wang (1997), when considering the roughness of the Earth's topography, both concludes that the series must be divergent in the strict, mathematical sense, but, from a practical point of view, divergence can be changed into convergence by a reasonable smoothing of the topography. In this context Moritz (1980) refers to the approximation theorem of Krarup-Runge. We will return to this question in our concluding remarks.

\section{Comparison with the topographic potential bias}

The topographic potential bias was defined by Sjöberg (2007) as the error in analytical continuation of the external potential inside the topographic masses, and only the error due to the fact that the potential is regarded as harmonic in a domain, where it is not (i.e. inside the topographic masses), is considered. This means, that some errors of the spherical harmonic series above, such as those due to truncation and non-convergence, are not considered. For a constant topographic density Sjöberg (2007), (2009a) and (2009c) proved that the topographic bias for the total geoid height is given by the simple formula

$$
\delta N_{\text {bias }}(\theta, \lambda)=\frac{2 \pi \mu}{\gamma}\left[H^{2}(\theta, \lambda)+\frac{2 H^{3}(\theta, \lambda)}{3 R}\right]
$$

If the analytical continuation of the external potential to sea level is truncated to wavelengths of a maximum degree of $n_{\max }$, the corresponding contribution to the topographic bias becomes 
accordingly (Sjöberg 2007)

$$
\begin{aligned}
& \delta N_{\text {bias }}(\theta, \lambda)= \\
& \frac{2 \pi \mu}{\gamma} \sum_{n=0}^{n_{\max }} \sum_{n=-n}^{n}\left[\left(H^{2}\right)_{n m}+\frac{2\left(H^{3}\right)_{n m}}{3 R}\right] Y_{n m}(\theta, \lambda) .
\end{aligned}
$$

Comparing this result with Eq. (9) we notice that the topographic bias is exactly the first two terms of DWC error of the spherical harmonics, but the remaining terms of the latter error are now missing. Some further details of the difference between the two error concepts can be found in the discussion between Vermeer (2007) and Sjöberg (2008).

[A practical consequence of the topographic bias, in contrast to the DWC, is that the geoid height can be determined without applying the so-called terrain corrections (Sjöberg 2009b). However, this topic is outside the scope of this article.]

\section{Numerical studies}

The aim of the numerical study is to compare the downward continuation error/analytical continuation error and the topographic potential bias for the Earth Gravitational Model EGM08 (for an equivalent EGM) complete to degree and order 2160, corresponding to a resolution of the order of $5^{\prime}$. To do so we will use the global Digital Terrain Model 2006 (DTM2006) available as a series of spherical harmonics to the same degree and order (Pavlis et al. 2006).

Figure 1 shows the downward continuation error for geoid height estimated by Eq. (4a), with coefficients determined by Eqs. (4b) and (5). It is an interesting notation that small negative errors (down to about $-0.078 \mathrm{~m}$ ) occur in $33.5 \%$ and $1.5 \%$ of points of over the oceans and continents, respectively. The continental areas with negative DWC error are primarily located along the shorelines. The reason of the negative values may be due to the different natures of the truncation errors of the external and internal series of the DWC error. For comparison, Figure 2 illustrates the topographic potential bias determined by Eq. (10), which, in contrast to Figure 1 , shows only non-negative biases.

The difference of the downward continuation error minus the topographic bias, depicted in Figure 3 shows some significant differences (down to $-0.205 \mathrm{~m}$ ), mainly in high-elevated areas.

Table 1 contains some basic statistics in comparing the analytical continuation error (DWC) and the topographic bias. As said above, it can be seen that the bias is large in mountainous areas. The maxima (occurring in the Himalayas) of DWC error and topographic bias are 5.30 and 5.15 metre, respectively. The maximum topographic bias occurs for the topographic height of $6679 \mathrm{~m}$. The table shows also that the analytical continuation error and topographic bias are more or less the same, because of the same mathematical nature and physical concept. The statistic of the differences shows that the mean is zero and differences are small.

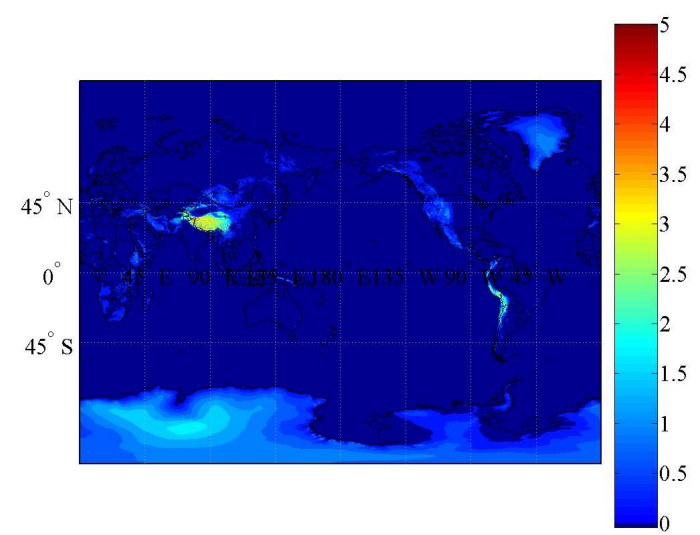

Figure 1. Downward continuation error for geoid height with a resolution 5'×5'. (Unit: $\mathrm{m}$ )

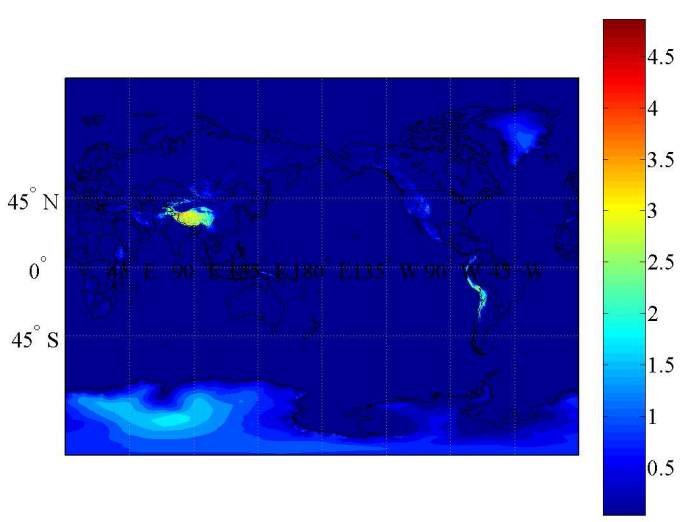

Figure 2. Topographic bias for geoid height with a resolution $5^{\prime} \times 5^{\prime}$. (Unit: $\mathrm{m}$ )

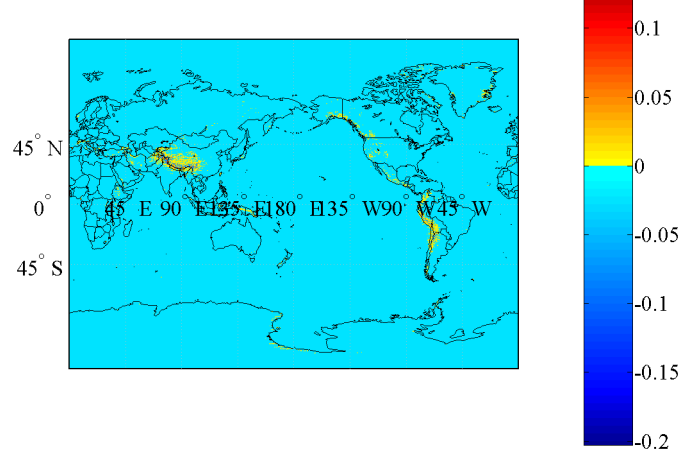

Figure 3. Difference between DWC error and topographic bias for geoid height with a resolution $5^{\prime} \times 5^{\prime}$. (Unit: $\mathrm{m}$ ) 
Table 1. Statistic of the DWC error and topographic bias for geoid height with a resolution $5^{\prime} \times 5^{\prime}$. (Unit: $\mathrm{m}$ )

\begin{tabular}{ccccc}
\hline & Max & Mean & Min & Std. \\
\hline \hline DWC error (Sjöberg, 1977) & 5.300 & 0.101 & -0.079 & 0.317 \\
\hline Topographic bias (Sjöberg, 2007) & 5.153 & 0.101 & 0 & 0.317 \\
\hline Difference & 0.185 & 0 & -0.205 & 0.004
\end{tabular}

The degree variances of the DWC error and the topographic bias are further compared in Figures 4 and 5. Both figures show that the errors are dominated by their low-degree components. (Note that the vertical scale in Figure 4 is logarithmic.) However, as can be seen in Figure 5, after about degree 100 the cumulative rms errors differ significantly and increase very slowly towards $0.253 \mathrm{~m}$ and $0.256 \mathrm{~m}$ for degree 2160 . Although the two errors agree well for low- and medium-degree components, their difference increases with the degree (see Figures 6 and 7).

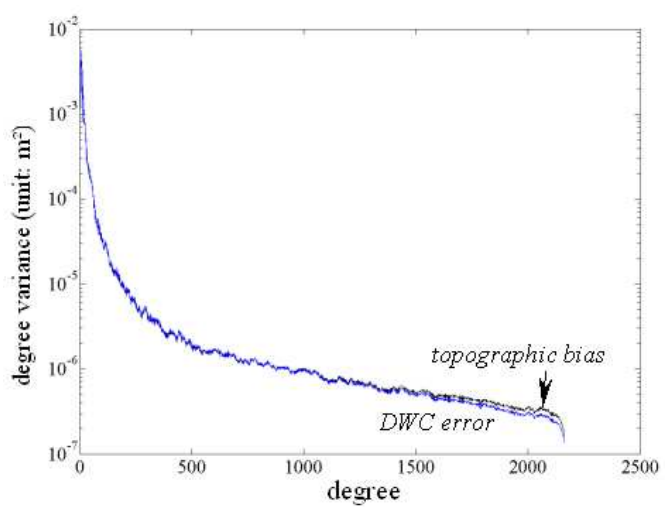

Figure 4. Comparison between DWC error and topographic bias degree variances up to 2160 degree. (Unit: $\mathrm{m}^{2}$ )

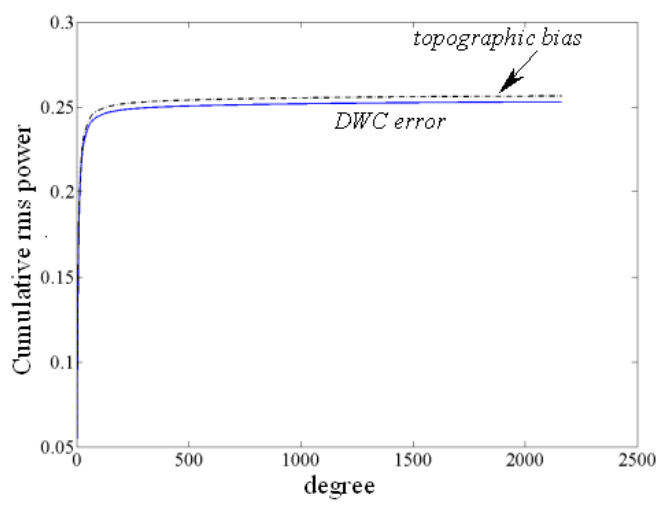

Figure 5. Cumulative global rms errors up to degree 2160. (Unit: m)

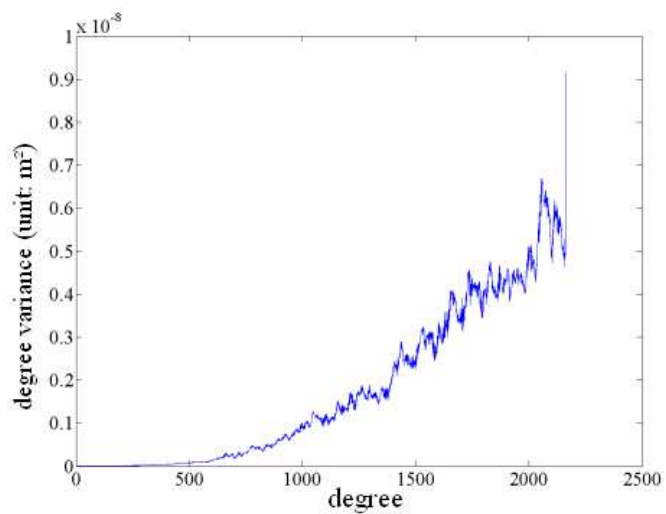

Figure 6. Degree variances of the difference between DWC error and topographic bias to 2160 degree. (Unit: $\mathrm{m}^{2}$ )

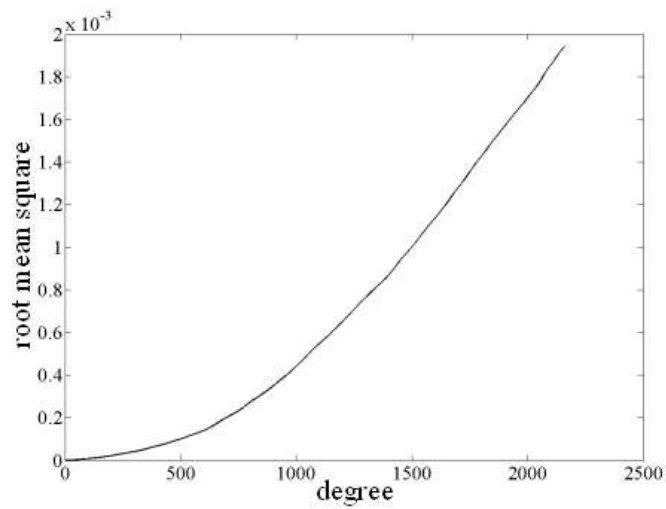

Figure 7. The global rms difference per degree for the two types of errors to degree 2160. (Unit: $\mathrm{m}$ )

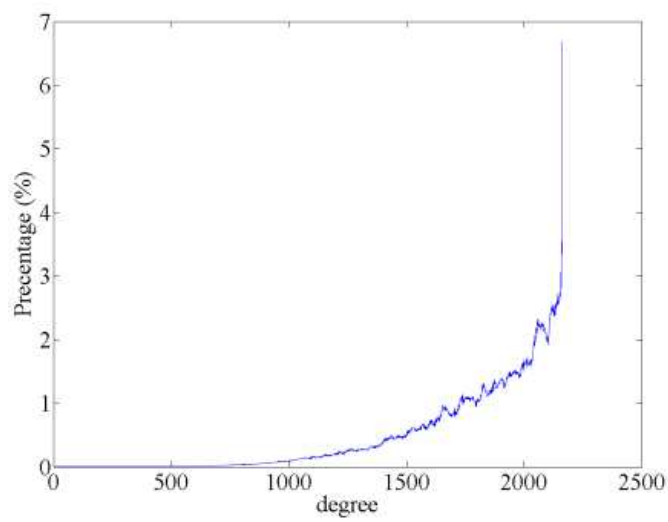

Figure 8. Relative degree variances of the difference of DWC error and topographic bias with respect to the degree variances of topographic bias to degree 2160. (Unit: Percent) 


\section{Journal of Geodetic Science}

Moreover, as can be seen from Figure 8, the relative difference grows quickly with the degree after, say, degree 2000. Hence, we may expect the DWC error to deviate considerably from the topographic bias for ultra-high degree harmonic expansions of the gravity field. This diverging pattern we refer to the shape of the DWC error, as the harmonic series of the topographic bias cannot be divergent.

\subsection{The zero- and first-degree contributions}

From Eq. (3) follows that the zero- and first-degree contributions to the DWC error can be written

$$
\delta N_{0}=2 \pi \frac{\mu}{\nu}\left[\left(H^{2}\right)_{0}+\frac{2}{3} \frac{\left(H^{3}\right)_{0}}{R}\right]
$$

and

$$
\delta N_{1}(\theta, \lambda)=2 \pi R^{2} \frac{\mu}{\nu} \sum_{m=-1}^{1} A_{1 m} Y_{1 m}(\theta, \lambda) .
$$

This shows that despite of that EGM08 (as is custom for EGMs) is defined in such a way that the zero- and first-degree harmonics are zero, the geoid heights provided by this model will include such harmonics (see also Sjöberg 2001).

For comparison, the topographic potential bias, computed by Eq. (11), and the DWC error, given by Eq. (12), yield the same zero-degree error of $5.3 \mathrm{~cm}$, but the first-degree harmonics differ slightly: the rms of the first degree dwc error and topographic bias are both $0.3 \mathrm{~cm}$.

Table 2. Comparison of the low-degree and -order terms of coefficients $A_{n m}$. In order to get the spectral DWC error of geoid height, each coefficient should be multiplied by the factor $\mu R^{2} / \gamma=7.37 \times 10^{5} \mathrm{~m}$.

\begin{tabular}{cccccccc}
\hline degree order & \multicolumn{2}{c}{ Coefficients $A_{n m}$} & degree order & \multicolumn{2}{c}{ Coefficients $A_{n m}$} \\
\hline $\mathrm{n}$ & $\mathrm{m}$ & cos part & sin part & $\mathrm{n}$ & $\mathrm{m}$ & cos part & sin part \\
\hline \hline 0 & 0 & $7.07 \times 10^{-08}$ & $0.00 \times 10^{0}$ & 4 & 0 & $2.55 \times 10^{-08}$ & $0.00 \times 10^{0}$ \\
\hline 1 & 0 & $-3.21 \times 10^{-09}$ & $0.00 \times 10^{0}$ & 4 & 1 & $-3.61 \times 10^{-10}$ & $-5.16 \times 10^{-08}$ \\
\hline 1 & 1 & $1.34 \times 10^{-08}$ & $3.03 \times 10^{-08}$ & 4 & 2 & $-3.66 \times 10^{-08}$ & $3.21 \times 10^{-09}$ \\
\hline 2 & 0 & $4.36 \times 10^{-08}$ & $0.00 \times 10^{0}$ & 4 & 3 & $8.95 \times 10^{-09}$ & $-4.03 \times 10^{-08}$ \\
\hline 2 & 1 & $-2.63 \times 10^{-09}$ & $3.08 \times 10^{-08}$ & 4 & 4 & $1.74 \times 10^{-08}$ & $1.24 \times 10^{-08}$ \\
\hline 2 & 2 & $-3.71 \times 10^{-08}$ & $4.81 \times 10^{-09}$ & 5 & 0 & $-5.73 \times 10^{-08}$ & $0.00 \times 10^{0}$ \\
\hline 3 & 0 & $-6.57 \times 10^{-08}$ & $0.00 \times 10^{0}$ & 5 & 1 & $7.62 \times 10^{-09}$ & $-7.47 \times 10^{-09}$ \\
\hline 3 & 1 & $-3.23 \times 10^{-10}$ & $3.26 \times 10^{-08}$ & 5 & 2 & $1.61 \times 10^{-09}$ & $-7.35 \times 10^{-09}$ \\
\hline 3 & 2 & $-4.29 \times 10^{-08}$ & $1.33 \times 10^{-08}$ & 5 & 3 & $3.90 \times 10^{-09}$ & $-3.46 \times 10^{-08}$ \\
\hline 3 & 3 & $-6.18 \times 10^{-09}$ & $-6.80 \times 10^{-09}$ & 5 & 4 & $+3.42 \times 10^{-08}$ & $-1.98 \times 10^{-08}$ \\
\hline & & & & & & &
\end{tabular}

In Table 2 we illustrate the lowest degree and order terms of coefficients $A_{n m}$. They can be converted to geoid height components by multiplying by the factor $\mu R^{2} / \nu \approx 7.37 \times 10^{5} \mathrm{~m}$ (computed for topographic density set to $2.67 \mathrm{~g} \mathrm{~cm}^{-3}$ and normal gravity $981 \mathrm{Gal}$ ). By subtracting these coefficients from those of the EGM08 potential coefficients, the latter model is converted to the coefficients of the disturbing potential at sea-level (inside the topography over continents). (The full set of coefficients can be obtained from the authors). As mentioned, this set of coefficients can contribute to a correction of the geoid computed by EGM08 of as much as $5.3 \mathrm{~m}$.

\section{Concluding remarks}

This study shows that the so-called DWC error in applying EGM08 to geoid determination is large in regions with high topography, ranging to $5.15 \mathrm{~m}$ in the Himalayas. The DWC error differs from the topographic potential bias, caused by the use of a harmonic series within the topographic masses, where the potential is not harmonic. While the topographic bias is caused solely by the analytical continuation into the masses, the DWC error also has other sources. For the EGM08 the two errors differ within -0.20 and $+0.18 \mathrm{~m}$, where the DWC error takes on the most extreme values.

According to Krarup-Runge's theorem (Moritz 1980, Ch. 7) the external spherical harmonic representation of the geopotential is practically convergent down to the Bjerhammar sphere (a sphere 
completely embedded in the Earth's interior), implying that the series will not experience any error (of arbitrarily chosen significance level) related with a divergent series as long as the series is truncated. This conclusion contradicts our discussions at the beginning of Sect. 2.1 and of the numerical results of Figures 7 and 8 in Sect. 4, which show clear divergent shapes of the error differences.

We showed also that the zero-degree error and rms first-degree error of the geoid height, when directly determined by EGM08, agree exactly to $5.3 \mathrm{~cm}$ and $0.3 \mathrm{~cm}$, respectively, for the DWC error and topographic error. Hence, despite of the fact that EGM08 is defined with vanishing zero- and first-degree terms, implying that the mass and center of the reference ellipsoid agree with the mass and gravity center of the Earth, the geoid will experience such harmonics. This effect is due to the fact that the geoid partly runs inside the topography.

The contents of this article are not only valid for applications with EGM08 alone, but they are also useful when combining an EGM with terrestrial gravity data. For instance, as pointed out by Sjöberg (2005), in geoid computation by the well-known remove-compute-restore technique the EGM should be corrected accordingly.

\section{References}

Ågren J., 2004, The analytical continuation bias in geoid determinatoin using potential coefficients and terrestrial garvity data. J. Geod., 78:314-332.

Jekeli Ch., 1981, The downward continuation to the Earth's surface truncated spherical and ellipsoidal harmonic series of the gravity and height anomalies. Rep 323, Department of Geodetic Science, The Ohio State University, Columbus.

Moritz H., 1980, Advanced Physical Geodesy, H Wichmann Verlag, Karlsruhe.

Pavlis N.K., Holmes S.A., Kenyon S.C., Factor J.K., 2008, An Earth Gravitational Model to degree 2160: EGM2008, Presented at the EGU General Assembly, Vienna, Austria, April 13-18, 2008.

Pavlis N., Factor K., Holmes Simon A., 2006, Terrain-Related Gravimetric Quantities Computed for the Next EGM. Presented at the $1^{\text {st }}$ International symposium of the International gravity service 2006, August 28- September 1, Istanbul, Turkey.

Sjöberg L.E., 1977, On the errors of spherical harmonic develop- ments of gravity at the surface of the Earth. Rep 257, Department of Geodetic Science, The Ohio State University, Columbus.

Sjöberg L.E., 1980, On the convergence problem for the spherical harmonic expansion of the geopotential at the surface of the Earth. Boll. Geod. Sci. Aff., 39(4):261-271.

Sjöberg L.E., 2000, Topographic effects by the Stokes-Helmert method of geoid and quasi-geoid determinations. J. Geod., 74:255-268.

Sjöberg L.E., 2001, Topographic and atmospheric corrections of the gravimetric geoid determination with special emphasis of the effects of degrees zero and one. J. Geod.,75:283-290.

Sjöberg L.E., 2005, A discussion on the approximations made in the practical implementation of the remove-compute-restore technique in regional geoid modelling. J. Geod., 78:645-653.

Sjöberg L.E., 2007, The topographic bias by analytical continuation in physical geodesy. J. Geod. 81:345-350.

Sjöberg L.E., 2008, Answers to the comments by M. Vermeer on L. E. Sjöberg (2007) “The topographic bias by analytical continuation in physical geodesy". J. Geod., 82:451-452.

Sjöberg L.E., 2009a, On the topographic bias in geoid determination by the external gravity field. J. Geod., 83:967-972.

Sjöberg L.E., 2009b, The terrain correction in gravimetric geoid determination - is it needed? Geophys J. Int., 176:14-18.

Sjöberg L.E., 2009c, Solving the topographic bias as an Initial Value Problem. Art. Sat., 44(4):77-84.

Sun W., Sjöberg L.E., 2001, Convergence and optimal truncation of binomial expansions used in isostatic compensations and terrain corrections, J. Geod., 74:627-636.

Vermeer M., 2007, Comment on Sjöberg (2007) "The topographic bias by analytical continuation in physical geodesy" J. Geod.,81(5):345-350. J. Geod., 81:445-450.

Wang Y.M., 1997, On the error of analytical downward continuation of the earth's external gravitational potential on and inside the earth's surface. J. Geoid., 71:70-82. 